\title{
NON-COMMUTATIVE DUPLICATES OF FINITE SETS
}

\author{
CLAUDE CIBILS
}

\begin{abstract}
We classify the twisted tensor products of a finite set algebra with a two elements set algebra using colored quivers obtained through considerations analogous to Ore extensions. This provides also a classification of entwining structures between a finite set algebra and the grouplike coalgebra on two elements. The resulting 2-nilpotent algebras have particular features with respect to Hochschild (co)homology and cyclic homology.
\end{abstract}

\section{INTRODUCTION}

A geometric category can be studied through the category of commutative algebras of functions on the spaces with values in a a commutative ring or a field, where the functions have properties according to the geometrical nature of the spaces; both categories are usually equivalent through this contravariant functor. Using this equivalence the algebraic approach is a substitute for the geometric one, keeping the geometric origine as a background. In this context the tensor product of algebras corresponds to the product of the spaces. The non-commutative geometry approach uses non-commutative algebras as substitutes for the spaces, providing a setting corresponding to virtual non-commutative spaces.

The set algebra of $k$ valued functions over a a finite set $E$ is a commutative semi-simple basic finite dimensional $k$-algebra, isomorphic to $k \times \cdots \times k$ where the number of copies of $k$ equals the cardinality of $E$. The tensor product of algebras $k^{E} \otimes_{k} k^{F}$ corresponds to the cartesian product $E \times F$. The non-commutative counterpart consist on twisted tensor products of $k^{E}$ and $k^{F}$ corresponding to noncommutative virtual cartesian products of the sets $E$ and $F$. Recall that a twisted tensor product of two algebras is an algebra structure on the tensor product of the underlying vector spaces such that the natural inclusions of the original algebras are algebra maps. This structure has been considered by P. Cartier when lecturing in Paris, 5. Several authors have also considered such product in different contexts, see the work of J. Baez [1, A. Cap, H. Schichl, J. Vanžura [4 and G. Maltsiniotis [9]. Note that twisted tensor products between an algebra and the polynomial algebra in one variable corresponds precisely to Ore extensions, see for instance 10] for the definition and use of Ore extensions.

The problem of classifying structures arising this way is difficult. Quite diverse non semi-simple one parameter families of algebras can occur. In this paper we use the equivalence between finite sets and semi-simple basic algebras over a field $k$ in order to classify the non-commutative duplicates of a set. More precisely the usual duplicate of a set $E$ is the disjoint union of two copies of $E$, namely $E \times\{a, b\}$ which has algebra set

$$
k^{E \times\{a, b\}}=k^{E} \otimes k^{\{a, b\}}=k^{E} \oplus k^{E} .
$$


Our purpose in this paper is to concentrate in this case corresponding to noncommutative duplicates of a finite set $E$.

We recall first some facts concerning twisted tensor products of algebras and how they are obtained. Then we provide a classification of the twisted tensor products of $k^{E}$ and $k^{\{a, b\}}$ through the set of interlacings which provides an analogous setting to Ore extensions. The results shows that non-commutative duplicates of a finite set are provided by one-valued quivers with set of vertices $E$ which has even length oriented cycles if any and provided with a coloration. The corresponding non semisimple non-commutative 2-nilpotent algebras obtained through the path algebra construction produces the set of non-commutative duplicates of $E$.

This result also provides a classification of entwinings maps between the algebra $k^{E}$ and the two-dimensional grouplike coalgebra $k\{a, b\}$. Indeed, an entwining consists of an algebra, a coalgebra, and a map satisfying certain properties, see [3] or [2, Sec. 2.1]. In case the coalgebra is finitely generated and projective over the ground ring, there is a bijection between entwinings and interlacings between the algebra and the dual of the coalgebra, see [2. Theorem 8]. Finalley notice that $k^{\{a, b\}}$ is the dual of the grouplike coalgebra $k\{a, b\}$.

In the last section we study the Hochschild cohomology of the non-commutative duplicates of a finite set $E$. The main result is that the Hochschild cohomology is finite dimensional if and only if the quiver associated to the situation has no oriented cycles other than loops, regardless of the coloration. In that case the only possible non zero vector spaces lies in degrees 0 and 1 .

We also compute cyclic homology of this algebras in characteristic zero showing that the dimension is constant in even degrees. In odd degrees, the dimension of the cyclic homology counts the number of connected components having a proper circuit of length dividing the degree augmented by one one.

Concerning Hochschild homology, we obtain that its dimension is finite if and only if the quiver has no oriented cycles, in which case all the homology vector spaces vanishes except in degree 0 where the dimension equals the dimension of the even cyclic homology.

\section{Twisted TENSOR PRODUCTS AND INTERLACINGS}

Let $k$ be a field. We consider $k$-algebras which are associative and unitary algebras over $k$. We recall some basic facts concerning twisted products of algebras.

Let $A$ and $B$ be $k$-algebras and let $A \otimes_{k} B$ be the tensor product of the underlying $k$-vector spaces. A twisted tensor product algebra of $A$ and $B$ is a $k$-algebra structure on $A \otimes_{k} B$ such that the canonical inclusions $a \mapsto a \otimes 1$ and $b \mapsto 1 \otimes b$ are $k$-algebra maps. Note that we do not assume any extra structure or possible actions on the original algebras which could provide the algebra structure of the twisted tensor product. Of course an action or a grading of a group on an algebra provide examples of twisted products, see 2.6

Lemma 2.1. Let $\Lambda=A \otimes_{k} B$ be a twisted tensor product algebra. Then

$$
(a \otimes 1)(1 \otimes b)=a \otimes b .
$$

Proof The product map $m: \Lambda \otimes_{k} \Lambda \rightarrow \Lambda$ is a $\Lambda$-bimodule map and the restriction to $((A \otimes 1) \otimes(1 \otimes B))$ is an $A-B$ - bimodule map. Moreover $((A \otimes 1) \otimes(1 \otimes B))$ is a free $A-B$ bimodule generated by $((1 \otimes 1) \otimes(1 \otimes 1))$ and we have $m((1 \otimes 1) \otimes(1 \otimes 1))=$ 
$(1 \otimes 1)$. Consequently

$$
\begin{aligned}
m((a \otimes 1) \otimes(1 \otimes b)) & =m(a(1 \otimes 1) \otimes(1 \otimes 1) b) \\
& =a((1 \otimes 1) \otimes(1 \otimes 1)) b \\
& =a m(1 \otimes 1) b \\
& =a \otimes b .
\end{aligned}
$$

As a consequence the structure of a twisted product is determined by the value of the products of elements of $B$ with elements of $A$. Let $\tau: B \otimes A \rightarrow A \otimes B$ be the map given by $\tau(b \otimes a)=(1 \otimes b)(a \otimes 1)$, in other words $\tau$ provides the product of $b$ and $a$ inside the twisted tensor product of algebras.

Proposition 2.2. The map $\tau$ verifies $\tau(b \otimes 1)=1 \otimes b, \tau(1 \otimes a)=a \otimes 1$ and the braiding conditions, namely the diagrams below commute (tensor signs are omitted or replaced by commas)

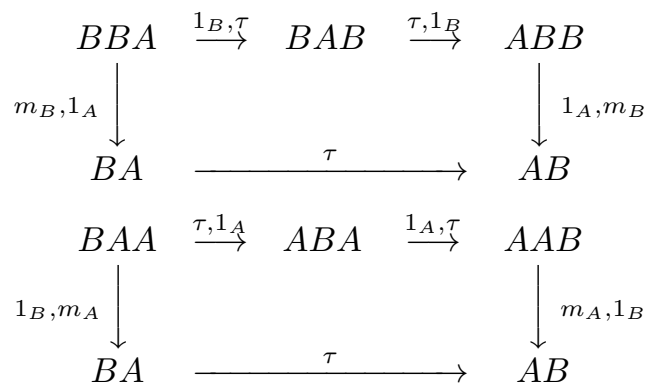

Proof: The first diagram correspond to the associativity condition of the product for triples $\left(b, b^{\prime}, a\right)$ namely $(b \otimes 1)\left(b^{\prime} \otimes 1\right)(a \otimes 1)$ while the second corresponds to triples $\left(b, a, a^{\prime}\right)$.

Definition 2.3. An interlacing is a linear map map $\tau: B A \longrightarrow A B$ verifying the preceeding properties.

Note that an interlacing is also called a factorization structure, see for instance 8]. The following result is now obvious :

Theorem 2.4. Let $A$ and $B$ be $k$-algebras. The twisted tensor product structures on $A \otimes_{k} B$ are in one to one correspondence with interlacings.

Example 2.5. The usual tensor product of algebras is obtained through the trivial interlacing, namely the flip map $\tau(b \otimes a)=a \otimes b$.

Example 2.6. Let $G$ be a group acting by automorphisms on a $k$ algebra $A$ and let $\tau$ be the map given by $\tau(s \otimes a)=s(a) \otimes s$. Then $\tau$ is an interlacing which provides the well known skew group algebra $A[G]$. As usual this can be generalized, replacing $k G$ by a Hopf algebra $H$ and the action by an $H$-module algebra structure on $B$.

Our purpose is to investigate the set of twisted tensor products between two algebras in order to provide a classification and properties relatively to the original algebras. As quoted in the Introduction, in this paper we will consider the case where $B=k^{\{a, b\}}$ is the set algebra of two elements $\{a, b\}$. Note that the trivial interlacing provides the usual tensor product $A \otimes k^{\{a, b\}}=A \oplus A$. Note also that the 
two elements algebra $k^{\{a, b\}}$ is isomorphic to the algebra of truncated polynomials $k[X] /\left(X^{2}-X\right)$ where $a$ correspond to $X$ and $b$ to $1-X$.

In order to classify interlacings we consider the following set.

Definition 2.7. Let $Y_{A}$ be the set of couples $(f, \delta)$ such that $f$ is an endomorphism of $A$ and $A \stackrel{\delta}{\longrightarrow}{ }^{f} A$ is an idempotent derivation verifying

$$
f=f^{2}+\delta f+f \delta .
$$

Remark 2.8. The notation ${ }^{f} A$ stands for the A-bimodule given by the vector space A with left action modified by $f$, namely $a \cdot a^{\prime}=f(a) a^{\prime}$. The right action is the standard one given by the product of $A$.

Remark 2.9. The data above can be interpreted as a sort of Ore extensions associated to the quotient of the polynomial algebra in one variable that we consider. One can prove that Ore extensions (see for instance [10]) are precisely twisted tensor products of a $k$-algebra with the polynomial algebra in one variable $k[x]$. The proof of this fact can be performed following the lines of the next Proposition.

Proposition 2.10. The set $Y_{A}$ is in one-to-one correspondence with the set of interlacings between the two elements set algebra $k[X] /\left(X^{2}-X\right)$ and a $k$-algebra A.

Proof: Consider the $k$-module $A \otimes\left[k[X] /\left(X^{2}-X\right)\right]=A[X] /\left(X^{2}-X\right)$. An interlacing $\tau$ is determined by the values $\tau(X, a)$ corresponding to the product $X a$. We put $\tau(X, a)=X a=\delta(a)+f(a) X$. The braiding conditions provides the equalities $X(X a)=X a$ and $X(a b)=(X a) b$. The first one translates into $\delta^{2}=\delta$ and $f=f^{2}+\delta f+f \delta$ while the second provides that $f$ is an endomorphism of $A$ and $\delta$ is a derivation with coefficients in ${ }^{f} A$. Conversely it is clear that those conditions insures that the map $\tau$ defined by the above formulae is an interlacing. Note that in this setting the trivial interlacing corresponds to the identity endomorphism and the zero derivation.

Remark 2.11. One could expect a cohomological interpretation of $Y_{a}$. It appears that $Y_{A}$ do not seem to have any additional structure, in particular no natural composition law of elements of $Y_{A}$ seem to exist. In the next section it will become clear that interior derivations do not provide in general trivial tensor twisted products.

Definition 2.12. The set of 2-interlacings of an algebra $A$ is the set of interlacings between $A$ and $k[X] /\left(X^{2}-X\right)$. We have proved that this set is in one-to-one correspondence with $Y_{A}$.

\section{Set Algebras And 2-INTERLacingS}

Our first purpose in this section is to describe $Y_{k^{E}}$ where $k^{E}$ is the set algebra of a finite set $E$. The second purpose is to classify the family of twisted tensor algebras obtained through the 2-interlacings. Recall that $k^{E}$ is the $k$-algebra of functions on $E$, namely $k^{E}=\{a: E \longrightarrow k\}$ where the product is given by $\left(a a^{\prime}\right)(x)=$ $a(x) a^{\prime}(x)$. Of course $k^{E}$ is isomorphic to the vector space with basis the set $E$ and componentwise product

$$
\left(\sum_{x \in E} a_{x} x\right)\left(\sum_{x \in E} a_{x}^{\prime} x\right)=\sum_{x \in E}\left(a_{x} a_{x}^{\prime}\right) x .
$$


In other words $k^{E}$ is a product of copies of $k$ indexed by $E$, and $E$ is a complete set of primitive orthogonal idempotents of $k^{E}$.

It is well known that $A=k^{E}$ is cohomologicaly trivial, which means that the Hochschild cohomology of $A$ with coefficients in any $A$-bimodule vanish in positive degrees. In particular any derivation is interior, this fact will provide an exhaustive way for describing the set of 2-interlacings. For the convenience of the reader as well as in order to introduce notation we provide a proof of this result.

Let $A$ be a $k$-algebra and $\mathrm{M}$ an $A$-bimodule. A derivation is a $k$-linear map $\delta: A \rightarrow M$ such that $\delta\left(a a^{\prime}\right)=a \delta\left(a^{\prime}\right)+\delta(a) a^{\prime}$. An interior derivation is associated to each element $m$ of $M$ by the formula $\delta_{m}(a)=a m-m a$. One can check that interior derivations are indeed derivations.

The following result is well known and easy to prove, it provides the classification of $A$-bimodules when $A$ is a finite set algebra over a field.

Proposition 3.1. Let $k$ be a field, $E$ be a set and $A=k^{E}$ be the set algebra. Any finitely generated A-bimodule is isomorphic to a direct sum of simple modules. The complete list of simple modules up to isomorphism is $\left\{{ }_{v} k_{u}\right\}_{u, v \in E}$ where ${ }_{v} k_{u}$ is a one dimensional vector space with identity actions of $v$ on the left, of $u$ on the right, and zero actions of others set elements.

According to this Proposition it suffices to consider simple bimodule of coefficients in order to prove that any derivation is inner.

Proposition 3.2. Let $A=k^{E}$ be a set algebra, let ${ }_{v} k_{u}$ be a simple bimodule and let $\delta: A \longrightarrow{ }_{v} k_{u}$ be a derivation. If $u \neq v$ the derivation is inner. In case $u=v$ we have $\delta=0$.

Proof: We prove first that the space of derivations is one-dimensional if $u \neq v$, and is zero if $u=v$. Let $e \in{ }_{v} k_{u}$ be a fixed non zero element and for each $x \in E$ let $\lambda_{x} \in k$ defined by $\delta(x)=\lambda_{x} e$. We assert that $\lambda_{x}=0$ if $x \neq u$ or $x \neq v$. Indeed,

$$
\lambda_{x} e=\delta(x)=\delta\left(x^{2}\right)=x \delta(x)+\delta(x) x=\lambda_{x} x e+\lambda_{x} e x=0+0=0 .
$$

If $u=v$ we have $\lambda_{u} e=\delta(u)=2 \lambda_{u} e$ which implies $\lambda_{u}=0$. If $u \neq v$ then

$$
0=\delta(v u)=v \delta(u)+\delta(v) u=\lambda_{u} v e+\lambda_{v} e u=\left(\lambda_{u}+\lambda_{v}\right) e .
$$

We consider now the interior derivation $\delta_{e}$ given by $\delta_{e}(x)=x e-e x$. Of course we have $\delta_{e}(x)=0$ if $x \neq u$ or $x \neq v$. Assuming $u \neq v$, we obtain $\delta_{e}(v)=e$ while $\delta_{e}(u)=-e$. Consequently the space of interior derivations is also one dimensional and every derivation is inner.

Towards the description of the set of 2-interlacings, we recall that each algebra endomorphism of $k^{E}$ is determined by a set map $\varphi: E \longrightarrow E$. Actually this is a special case of the anti-equivalence between the category of finite sets and the category of semi-simple basic and commutative algebras. More precisely let $f$ be an algebra endomorphism of $k^{E}$. There exist a unique set map $\varphi: E \longrightarrow E$ such that for each $e \in E$ we have

$$
f(e)=\sum_{\{x \mid \varphi(x)=e\}} x .
$$

Lemma 3.3. Let $f$ an algebra endomorphism of $A=k^{E}$ given by a set map $\varphi: E \longrightarrow E$. Let $\delta: A \longrightarrow{ }^{f} A$ be a derivation. There exist $a \in A$ such that for each 
$e \in E$ we have $\delta(e)=(f(e)-e) a=\Sigma_{\varphi(x)=e} a_{x} x-a_{e} e$. Moreover

$$
\delta^{2}(e)=\sum_{\varphi(y)=e} a_{y} a_{\varphi(y)} y-\sum_{\varphi(x)=e} a_{x}\left(a_{x}+a_{e}\right) x+a_{e}^{2} e .
$$

Remark 3.4. The element $a$ of the Lemma is uniquely determined once normalized at loop elements of $E$, namely $a_{e}=0$ if $f(e)=e$. We call if the determining element of $\delta$.

Proof: Since $\delta$ is interior there exist $a$ such that $\delta(e)=e \cdot a-a_{e} e=f(e) a-a e$ $=(f(e)-e) a$. The fact that $a$ is unique follows at once from the previous considerations. Note also that if $u \neq v$ we have $H^{0}\left(A,{ }_{v} k_{u}\right)=0$.

Now

$$
\begin{aligned}
\delta^{2}(e) & =\sum_{\varphi(x)=e} a_{x} \delta(x)-a_{e} \delta(e) \\
& =\sum_{\varphi(x)=e} a_{x}\left(\sum_{\varphi(y)=x} a_{y} y-a_{x} x\right)-a_{e}\left(\sum_{\varphi(x)=e} a_{x} x-a_{e} e\right) \\
& =\sum_{\varphi(y)=e} a_{y} a_{\varphi(y)} y-\sum_{\varphi(x)=e} a_{x}\left(a_{x}+a_{e}\right) x+a_{e}^{2} e .
\end{aligned}
$$

In order to describe the idempotent derivations $\delta: A \longrightarrow{ }^{f} A$ it is useful to introduce the quiver of the endomorphism $f$ given by a set map $\varphi: E \longrightarrow E$. Recall that a quiver $Q$ is a finite oriented graph with set of vertices $Q_{0}$, set of arrows $Q_{1}$ and two maps $s, t: Q_{1} \longrightarrow Q_{0}$ providing each arrow with a source and a target vertex.

Definition 3.5. Let $f$ be an endomorphism of the set algebra $A=k^{E}$ given by a set map $\varphi$. The quiver $Q_{f}$ of $f$ has set of vertices $E$ and an arrow from $x$ to $\varphi(x)$ for each $x \in E$. Two arrows $b$ and $a$ are concatenated if $s(b)=t(a)$

Remark 3.6. Quivers obtained this way are precisely one-valued quivers, that is each vertex of the quiver is the source of exactly one arrow, accordingly to the definition of a set map.

Definition 3.7. An oriented cycle of a quiver is a sequence of concatenated arrow such that the source of the first arrow coincides with the target of the last one. Its length is the number of arrows involved. A loop is an oriented cycle of length one, namely an arrow with the same source and target vertices. An oriented cycle is proper if it is not the iteration of a strictly smaller length oriented cycle. A loop vertex is a vertex where a loop has its source and target vertices.

Each connected component of a one-valued quiver has precisely one proper oriented cycle, which can be a loop.

Let $R$ be a connected component of the quiver $Q_{f}$. Let $\delta: k^{E} \longrightarrow{ }^{f}\left(k^{E}\right)$ be a derivation with determining element $a \in k^{E}$. Our first purpose is to describe those $a$ such that $\delta^{2}=\delta$.

Lemma 3.8. Let $A=k^{E}$ be a finite set algebra, $f$ an endomorphism of $A$ with set map $\varphi$ and quiver $Q_{f}$ and let $A \longrightarrow{ }^{f} A$ be an idempotent derivation with determining element $a \in k^{E}$. Let $u \longrightarrow v$ be an arrow of $Q_{f}$ with $u \neq v$ where $v$ is a non loop 
vertex and such that there is no arrow backwards. Note that $u$ cannot be a loop vertex since $Q_{f}$ is one-valued. Then $a_{u}, a_{v} \in\{-1,0\}$ and $a_{u} a_{v}=0$.

Proof: At the vertex $v$ we have the formula

$$
0=\left(\delta^{2}-\delta\right)(v)=\sum_{\varphi_{y}^{2}=v} a_{y} a_{\varphi(y)} y-\sum_{\varphi(x)=v} a_{x}\left(a_{x}+a_{v}+1\right) x+a_{v}\left(a_{v}+1\right) v .
$$

The $v$-coefficient of this sum is $a_{v}\left(a_{v}+1\right)$, then $a_{v} \in\{0,-1\}$. The $u$-coefficient is $-a_{u}\left(a_{u}+a_{v}+1\right)$. If $a_{v}=-1$ then $a_{u}=0$. If $a_{v}=0$ then $a_{u} \in\{0,-1\}$.

Next we define pre-colorations of $Q_{f}$, which will correspond to idempotent derivations.

Definition 3.9. Let $Q_{f}$ be the quiver of an algebra endomorphism $f$ provided by a set map $\varphi: E \longrightarrow E$. A pre-coloration of its vertices is an element $a$ of $k^{E}$ verifying the following conditions:

(1) In case of a connected component of $Q_{f}$ reduced to a round trip quiver $u \cdot \rightleftarrows \cdot v$ the colors $a_{u}$ and $a_{v}$ verify either $a_{u}+a_{v}+1=0$ or $a_{u}=a_{v}=0$.

(2) For a connected component different from the round trip quiver:

(a) In case $e$ is a non loop vertex then $a_{e} \in\{0,-1\}$.

(b) For each arrow the product of the colors at its source and target is 0 .

(c) For a loop vertex the color is irrelevant, we normalize it at 0 .

Proposition 3.10. Let $A=k^{E}$ be a finite set algebra, $f$ an endomorphism of $A$ with set map $\varphi$ and quiver $Q_{f}$ and let $A \longrightarrow{ }^{f_{A}}$ be an idempotent derivation with determining element $a \in k^{E}$. Then a is a pre-coloration of $Q_{f}$ on this connected component.

Proof: We consider a connected component $R$ of $Q_{f}$. Assume first there is a loop vertex $e$ in $R$ and consider the equation $\left(\delta^{2}-\delta\right)(e)=0$. The coefficient of $e$ is $a_{e}^{2}-a_{e}\left(2 a_{e}+1\right)+a_{e}^{2}+a_{e}$ which is 0 for any value of $a_{e}$. If the connected component $R$ is reduced to $e$ we have that $a$ is a pre-coloration. Otherwise let $x$ such that $\varphi(x)=e$ and $x \neq e$. The coefficient of $x$ in the equation $\left(\delta^{2}-\delta\right)(e)=0$ is

$$
a_{x} a_{e}-a_{x}\left(a_{x}+a_{e}+1\right)=a_{x}\left(a_{x}+1\right) .
$$

Then $a_{x} \in\{0,-1\}$. In case $R$ do not contain the round trip quiver the conclusion follows from the preceding lemma.

If $R$ contains the round trip quiver having vertices $u$ and $v$ the equation $\left(\delta^{2}-\right.$ $\delta)(u)=0$ provides the $v$-coefficient

$$
-a_{v}\left(a_{v}+a_{u}+1\right)
$$

and $u$-coefficient

$$
a_{u} a_{v}+a_{u}\left(a_{u}+1\right)=a_{v}\left(a_{v}+a_{u}+1\right) .
$$

It follows that $a_{u}+a_{v}+1=0$ or $a_{u}=a_{v}=0$.

In case $R$ is not reduced to the round trip quiver there is an arrow arriving to $u$ or $v$ - we assume $u$ is this vertex without lost of generality - coming from a vertex $w$ which cannot be a loop vertex since each vertex is the source of exactly one arrow. For the same reason this arrow cannot be part of a round trip quiver. The Lemma applies and we infer $a_{w}, a_{u} \in\{0,-1\}$ and $a_{u} a_{w}=0$. If $a_{u}=0$ the equations of the round trip quiver above provides $a_{v}=-1$ or $a_{v}=0$. If $a_{u}=-1$ then $a_{v}=0$. In both cases we obtain a pre-coloration. 
Remark 3.11. From the proof of the result it is clear that conversely a pre-coloration of $Q_{f}$ provides an idempotent derivation $k^{E} \longrightarrow{ }^{f}\left(k^{E}\right)$.

Finally we need to describe the pre-colorations corresponding to idempotent derivations verifying $f=f^{2}+\delta f+f \delta$.

Definition 3.12. A coloration of $Q_{f}$ is an element $a=\sum_{x \in E} a_{x} x \in k_{E}$ such that

(1) For a connected component reduced to the round trip quiver $u \cdot \rightleftarrows \cdot v$ the coefficients $a_{u}$ and $a_{v}$ verify $a_{u}+a_{v}+1=0$.

(2) For other connected components:

(a) In case $e$ is a non loop vertex, $a_{e} \in\{0,-1\}$

(b) For each arrow having no loop vertex target, one extremity value is 0 and the other is -1 .

(c) At a loop vertex the value of $a$ is irrelevant, we normalise it at 0 .

Note that a coloration is a pre-coloration.

Remark 3.13. Not any quiver $Q_{f}$ admits a coloration, clearly the length of non loop proper oriented cycles has to be even. For a connected component having even length proper oriented cycles, precisely two colorations exists. Otherwise a coloration of a connected component with a loop is completely determined by providing 0 and -1 values on the loop related vertices, namely on source of arrows having a loop target vertex.

Theorem 3.14. Let $E$ be a finite set, $A=k^{E}$, and let $f$ be an endomorphism of $A$ corresponding to a set map $\varphi$ with quiver $Q_{f}$. Let $a \in k^{E}$ be a normalized element (i.e. it has 0 value at loop vertices) ant let $\delta: k^{E} \longrightarrow{ }^{f}\left(k^{E}\right)$ be the inner derivation determined by a. The couple $(f, \delta)$ is a 2-interlacing for $A$ if and only if $a$ is a coloration of $Q_{f}$.

Proof: We already know that pre-colorations translates the fact that $\delta^{2}=\delta$. The condition $f=f^{2}+\delta f+f \delta$ provides the following for each $e \in E$ :

$$
\sum_{\varphi^{2}(y)=e}\left(a_{y}+a_{\varphi(y)}+1\right) y-\sum_{\varphi_{x}=e}\left(a_{e}+a_{x}+1\right) x=0 .
$$

Let $u \longrightarrow v$ be an arrow between different non loop vertices. The $u$-coefficient in the above equation for $v$ provides $a_{v}+a_{u}+1=0$. Since we have $a_{u}, a_{v} \in\{0,-1\}$ we infer that $a_{u} \neq a_{v}$. In case $v$ is a loop vertex the $u$-coefficient in the equation for $v$ gives

$$
\left(a_{u}+a_{v}+1\right)-\left(a_{v}+a_{u}+1\right)=0
$$

and there no restriction is inferred for $a_{v}$. The $v$-coefficient for the equation of $v$ provides

$$
\left(a_{v}+a_{v}+1\right)-\left(a_{v}+a_{v}+1\right)=0 .
$$

Finally assume that $R$ is the round trip quiver $u \rightleftarrows v$. The $u$-coefficient for the $v$-equation gives

$$
\left(a_{v}+a_{u}+1\right)=0
$$

which implies that the option $a_{u}=a_{v}=0$ of a pre-coloration do not provide an interlacing. In contrast the condition $a_{v}+a_{u}+1=0$ is maintained.

Theorem 3.15. Let $A=k^{E}$ be the algebra of a finite set $E$. The set $Y_{A}$ of 2interlacings of $A$ is in bijection with one-valued quivers on the set $E$ provided with a coloration. 


\section{Non-COmmutative Duplicates}

The aim of this section is to classify the twisted tensor products $k^{E} \otimes k^{\{a, b\}}$ where $E$ is a finite set. We will obtain a family of non semi-simple, non-commutative algebras, with square zero Jacobson radical.

From the preceding section it appears that we can assume that the quiver $Q_{f}$ of an endomorphism $f: k^{E} \longrightarrow k^{E}$ is connected. More precisely let $(f, \delta)$ be an endomorphism of $k^{E}$ and let $\delta$ be a derivation

$$
\delta: k^{E} \longrightarrow{ }^{f}\left(k^{E}\right)
$$

verifying $\delta^{2}=\delta$ and $f=f^{2}+\delta f+f \delta$. Let $k^{E} \otimes_{(f, \delta)}\left[k[X] /\left(X^{2}=X\right)\right]$ be the corresponding twisted tensor product.

Let $E^{1}, \ldots, E^{n}$ be the partition induced on $E$ by the connected components of $Q_{f}$ and let $f_{i}$ be the corresponding endomorphism of $k^{E_{i}}$. Clearly $\delta$ decomposes into $\delta_{i}: k^{E_{i}} \longrightarrow f^{f_{i}}\left(k^{E_{i}}\right)$ and we have

$$
k^{E} \otimes_{(f, \delta)} k[X] /\left(X^{2}-X\right)=\prod_{i=1, \cdots, n}\left(k^{E_{i}} \otimes_{\left(f_{i}, \delta_{i}\right)}\left[k[X] /\left(X^{2}-X\right)\right]\right) .
$$

First we consider the trivial case of a one element set $E$, the map $f$ is the identity, the quiver $Q_{f}$ is a loop and $\delta=0$. The only twisted tensor product of $k^{E}$ with $k[X] /\left(X-X^{2}\right)$ is the trivial one. There is no non trivial duplicate of a one element set.

The case where $E$ is an arbitrary finite set but $f$ is the identity reduces to preceding case, use for instance the connected components of $Q_{f}$ which are all of them loops, or perform the direct computation since the only derivation is the zero one.

Recall that for a quiver $Q$ the path algebra $k Q$ has a basis provided by all the oriented paths - that is finite sequences of concatenated arrows of $Q$ - which multiplies as they concatenate if this is possible, and have zero product otherwise. By definition the two sided ideal $\left\langle Q^{2}\right\rangle$ has a basis given by all the paths of length greater or equal 2. The quotient $k$-algebra $k Q /\left\langle Q^{2}\right\rangle$ has a basis provided by vertices and arrows. Its Jacobson radical has a basis given by the arrows and has zero square. It is well known that any finite dimensional, basic and split radical square zero algebra is obtained this way.

We assert that twisted tensor products $k^{E} \otimes\left[k[X] /\left(X^{2}-X\right)\right]$ are members of the preceding family of algebras. In order to prove this assertion we define a quiver related to a couple $(f, \delta) \in Y_{k^{E}}$. It will appear to be essentially the opposite quiver $Q_{f}^{\text {op }}$ of the set map $\varphi$ defining $f$, except for loop vertices which becomes two vertices.

Definition 4.1. Let $E$ be a finite set and $f: k^{E} \longrightarrow k^{E}$ be an algebra map provided by a set map $\varphi: E \longrightarrow E$ with quiver $Q_{f}$. Let $a$ be a coloration of $Q_{f}$ and let $\delta$ be the corresponding derivation. The related quiver $Q_{(f, \delta)}$ is obtained by replacing each connected component $R$ of $Q_{f}$ as follows

- If $R$ has no loops replace it by its opposite $R^{\text {op }}$ which has same set of vertices while arrows are reversed.

- If $R$ has a loop vertex $\ell$ (in which case $R$ do not contain other proper oriented cycles) remove the loop from $R^{\text {op }}$ and replace the vertex $\ell$ by two vertices $\ell_{0}$ and $\ell_{-1}$. Let $\varepsilon=0$ or -1 . Each arrow in $R^{\text {op }}$ from an $\varepsilon$ polarized vertex to $\ell$ is replaced by an arrow from the $\varepsilon$-polarized vertex to $\ell_{\varepsilon}$. Note that this procedure produces two connected components. 
Theorem 4.2. Let $E$ be a finite set and let $f: k^{E} \longrightarrow k^{E}$ be an algebra endomorphism given by a set map $\varphi$ with a connected quiver $Q_{f}$. Assume $Q_{f}$ is not the round trip quiver. Let $(f, \delta)$ be an interlacing of $k^{E}$ determined by a coloration a of $Q_{f}$. The twisted tensor algebra $k^{E} \otimes_{(f, \delta)}\left[k[X] /\left(X^{2}-X\right)\right]$ is isomorphic to the radical square zero algebra $k Q_{(f, \delta)} /\left\langle Q_{(f, \delta)}^{2}\right\rangle$.

Remark 4.3. Both algebras have the same dimension since $Q_{(f, \delta)}$ has more vertices but less arrows in the same quantity, namely the loops.

Proof: Note first that an algebra morphism

$$
\varphi: k Q_{(f, \delta)} \longrightarrow k^{E} \otimes_{(f, \delta)}\left[k[X] /\left(X^{2}-X\right)\right]
$$

is determined by a coherent choice of images of the vertices and the arrows of $Q_{(f, \delta)}$ since $k Q_{(f, \delta)}$ is a tensor algebra on the vertices set algebra of the arrows bimodule.

We define $\varphi(e)=e$ for non loop vertices of $Q_{f}$. In case of a loop vertex $\ell$ of $Q_{f}$ we put

$$
\varphi\left(\ell_{0}\right)=\ell X \text { and } \varphi\left(\ell_{-1}\right)=\ell(1-X)
$$

We have to verify that the set $(E \backslash\{\ell\}) \bigsqcup\{\ell X, \ell(1-X)\}$ is a complete set of orthogonal idempotents. Recall that $k^{E} \otimes_{(f, \delta)} k[X] /\left(X^{2}-X\right)$ is identified with $k^{E}[X] /\left(X^{2}-X\right)$ where the product is given by the twist $X b=\delta(b)+f(b) X$. More precisely if $e \in E$, we have

$$
X e=a(f(e)-e)+f(e) X=\sum_{\varphi(x)=e} a_{x} x-a_{e} e+\left(\sum_{\varphi(x)=e} x\right) X
$$

If $e$ is -1 -polarised, then

$$
X e=e+\left(\sum_{\varphi(x)=e} x\right) X
$$

If $e$ is 0 -polarised

$$
X e=\left(\sum_{\varphi(x)=e} x\right)(-1+X) .
$$

Finally if $e=\ell$ is a loop vertex, then

$$
X \ell=-\sum_{\varphi(x)=\ell a_{x}=-1} x+\left(\sum_{\varphi(x)=\ell} x\right) X
$$

Note that

$$
(\ell X)^{2}=\ell X \ell X=\ell\left(-\sum_{\varphi(x)=\ell a_{x}=-1} x+\left(\sum_{\varphi(x)=\ell} x\right) X\right) X=\ell X
$$

since $\varphi(x)=\ell$ and $\ell$ belongs to the sum $\sum_{\varphi(x)=\ell} x$.

Similarly $[\ell(1-X)]^{2}=\ell(1-X)$. We also have that $[\ell X][\ell(1-X)]=0=$ $[\ell(1-X)][\ell X]$ since $\ell X \ell(1-X)=\ell X(1-X)=\ell X-\ell X=0$.

Finally note that for a vertex $e \neq \ell$ we have $e \ell X=0$ and $\ell X e=0$ for both possible colorations of $e$ since

$$
1=\sum_{e \in E \backslash\{\ell\}} e+\ell X+\ell(1-X)
$$


We consider now arrows of $Q_{(f, \delta)}$. For $e$ a non loop vertex and $x \in E$ such that $\varphi(x)=e$ let ${ }_{x} a_{e}$ be the corresponding arrow. We assert that $\varphi\left({ }_{x} a_{e}\right)=x X e$ is a coherent choice since $\varphi\left({ }_{x} a_{e}\right)=\varphi\left(x_{x} a_{e} e\right)=\varphi(x) \varphi\left({ }_{x} a_{e}\right) \varphi(e)$. For $\varepsilon=0$ or -1 let $x$ be a $\varepsilon$-polarized vertex of $E$ such that $\varphi(x)=\ell$ and let ${ }_{x} a_{\ell_{\varepsilon}}$ be the corresponding arrow. We put

$$
\varphi\left({ }_{x} a_{\ell_{\varepsilon}}\right)=x X \ell(1+\varepsilon X) .
$$

In order to prove that $\varphi$ is surjective note that for a non loop vertex $e$ we have $y X e=0$ if $\varphi(y) \neq e$. Moreover, if $\ell$ is a loop vertex and $y$ is not $\varepsilon$ polarized then $y X \ell(1+\varepsilon X)=0$.

Since

$$
X=1 X 1=\sum_{u, v \text { vertices of } Q_{(f, \delta)}} v X u
$$

we have that

$$
\varphi\left(\sum_{\text {arrows of } Q_{(f, \delta)}} a\right)=X .
$$

Of course the entire algebra $k^{E}$ is also in the image of $\varphi$, hence $\varphi$ is surjective. An easy computation shows that a path of length two of $Q_{(f, \delta)}$ has zero image. Since both algebras have the same dimension the surjective map induces an isomorphism.

Theorem 4.4. Let $E=\{u, v\}$ and let $f: k^{E} \longrightarrow k^{E}$ be an algebra endomorphism given by a set-map $\varphi$ with quiver $Q_{f}=u \cdot \leftrightarrows \cdot v$. Let $(f, \delta)$ be an interlacing of $k^{E}$ determined by a coloration $a=a_{u} u+a_{v} v$ of $Q_{f}$, that is $a_{u}+a_{v}+1=0$. The twisted tensor algebra $k^{E} \otimes_{(f, \delta)} k[X] /\left(X^{2}-X\right)$ is isomorphic to the radical square zero algebra $k Q_{f} /\left\langle Q_{f}^{2}\right\rangle$.

Remark 4.5. The result of the Theorem is independent of the coloration, in other words different colorations of the round trip quiver provides the same twisted tensor product.

Proof: The theory we have developed shows that the twisted tensor product is the algebra $k^{E}[X] /\left(X-X^{2}\right)$ where the interlacing is given by $X u=a_{v} v-a_{u} u+v X$ and $X v=a_{u} u-a_{v} v+u X$. Recall that $a_{u}+a_{v}+1=0$. Note also that a direct computation can be performed to show that the condition is necessary and sufficient in order to have a well defined associative product.

We assert that the vertices of the algebra are $u$ and $v$ while the arrows are $v X u$ and $u X v$. In other words we construct a surjective algebra map $\psi: k Q_{f} \longrightarrow$ $k^{E}[X] /\left(X-X^{2}\right)$. In order to prove this we have to show that $X$ is in the image of $\psi$. Indeed $X=u X u+v X v+v X u+u X v$, the last two terms are in the image of $\psi$ by construction while $u X u=-a_{u} u$ and $v X v=-a_{v} v$. Finally note that $v X u X v=0=u X v X u$ which shows that $\psi$ provides a surjective map between algebras of dimension 4 , consequently they are isomorphic.

It is well known and easy to prove that a complet invariant up to isomorphism of radical square zero algebras $k Q /<Q^{2}>$ is provided by the quiver $Q$. Of course this fact will be a crucial piece in order to classify the non-commutative duplicates of a finite set through the related quiver.

We already note that a connected one-valued quiver not reduced to a loop, which is not the round trip quiver and with even length oriented cycle has exactly two colorations. In case there is no loop the related quiver do not depend on the 
coloration. In contrast in case there is a loop the related quivers differs depending on the coloration.

As we quote before, the related quiver of a connected quiver containing a loop has two connected components. Both have precisely one sink vertex and all other vertices are one-valued. We call such quivers one-sink-one-valued, observe that a quiver reduced to a vertex belongs to this family.

This discussion proves the following

Theorem 4.6. Let $E$ be a finite set. The complete list up to isomorphism of non-commutative duplicates of $E$ are provided by the disjoint union of quivers as follows

- connected one-valued quivers with an even length proper oriented cycle,

- connected one-sink-one-valued quivers

such that the total number of vertices involved equals $|E|+L_{1}$ where $L_{1}$ is the number of connected one-sink-one-valued components (corresponding to the number of loops of the original quiver). The non-commutative duplicates are the radical square zero algebras obtained with the opposites of those quivers.

Note that different 2-interlacings can provide the same related quiver. Coloration switches on non loop connected component provides the same related quiver. In case the set map has no fixed points (that is the quiver $Q_{f}$ has no loops), the fiber of the related quiver is precisely described this way. Its cardinality is 2 raised to the number of such connected components.

\section{HochSCHILd (CO)homology AND CYCLIC HOMOLOGY}

In order to compute the Hochschild cohomology of non-commutative duplicates of a finite set, we will use the classification of the preceding section - we have shown that they are provided by a precise family of quivers and the corresponding radical square zero algebras. We will also use the main results from [6] where the dimensions of the Hochschild cohomology for these algebras are computed.

Let $Q$ be a connected quiver provided by a finite set of vertices $Q_{0}$, a finite set of arrows $Q_{1}$ and two maps $s, t: Q_{1} \rightarrow Q_{0}$ determining the source and the target of each arrow. Let $Q_{n}$ be the set of paths of length $n$ in $Q$, namely the sequences of $n$ concatenated arrows. We denote $(k Q)_{2}$ the quotient of the path algebra of $Q$ modulo the two sided ideal generated by $Q_{2}$.

The dimension of the Hochschild cohomology of $(k Q)_{2}$ involves the sets of parallel paths. More precisely two paths are said to be parallel if they share the same source and the same terminal vertices. In case $X$ and $Y$ are sets of paths, $X / / Y$ is the set of couples of parallel paths $(\alpha, \beta)$ where $\alpha \in X$ and $\beta \in Y$.

The following result is proved in 6. It concerns any connected quiver different from a crown, where a $c$-crown is the quiver with $c$ vertices cyclically labelled by the cyclic group of order $c$, and $c$ arrows $a_{0}, \ldots, a_{c-1}$ such that $s\left(a_{i}\right)=i$ and $t\left(a_{i}\right)=i+1$.

Theorem 5.1. Let $Q$ be a connected quiver which is not a crown. Then

$$
\begin{gathered}
\operatorname{dim} H H^{n}\left((k Q)_{2}\right)=\left|\left(Q_{n} / / Q_{1}\right)\right|-\left|\left(Q_{n-1} / / Q_{0}\right)\right| \text { in case } n \geq 2 \\
\operatorname{dim} H H^{1}\left((k Q)_{2}\right)=\left|\left(Q_{1} / / Q_{1}\right)\right|-\left|Q_{0}\right|+1
\end{gathered}
$$




$$
\operatorname{dim} H H^{0}\left((k Q)_{2}\right)=\left|\left(Q_{1} / / Q_{0}\right)\right|+1
$$

Remark 5.2. In 6] there is a one unity error in the computation of the dimension of the degree one Hochshchild cohomology.

The preceding Theorem has an important consequence, see Corollary 3.2 of []

Corollary 5.3. Let $Q$ be a connected quiver which is not a crown. The graded cohomology $\mathrm{HH}^{*}\left(\left(k Q_{2}\right)\right)$ is finite dimensional if and only if $Q$ has no oriented cycles.

Observe that in case of a one-valued quiver the set $\left|\left(Q_{n} / / Q_{1}\right)\right|$ for $n \geq 2$ is only originated by oriented cycles. More precisely an oriented cycle of length $n-1$ preceded by an arrow provides the set of couples in $\left|\left(Q_{n} / / Q_{1}\right)\right|$ for $n \geq 2$. Observe also that in case of a connected one-sink-one-valued quiver $\left|\left(Q_{n} / / Q_{1}\right)\right|=\emptyset$ for $n \geq 2$.

Theorem 5.4. Let $A$ be a non-commutative duplicate algebra of a finite set, given by a disjoint union of connected one-sink-one-valued quivers only. Equivalently, there is no oriented cycles other than loops in the quiver of the set map defining the non-commutative duplicate.

Then $H H^{n}(A)=0$ for $n \geq 2$, while $\operatorname{dim} H H^{0}(A)$ is the number of connected components of the quiver and $\operatorname{dim} H H^{1}(A)$ is the Euler characteristic of the underlying graph of $Q$.

Theorem 5.5. Let $A$ be a non-commutative duplicate algebra of a finite set provided by a 2-nilpotent algebra associated to a quiver which has an oriented cycle. Then the graded cohomology $H^{*}(A)$ is infinite dimensional.

Proof: The result is clear from the previous considerations, and from the fact that a crown has infinite dimensional cohomology, see Proposition 3.3 of $[6]$.

Finally we resume our results in order to make a direct link with the set $Y_{k^{E}}$ of 2-interlacings.

Theorem 5.6. Let $(f, \delta)$ be a 2-interlacing of a finite set $E$, namely $f$ is an endomorphism of $k^{E}$ provided by a set map $\varphi: E \rightarrow E$ and $\delta: k^{E} \rightarrow{ }^{f}\left(k^{E}\right)$ is an idempotent derivation verifying $f=f^{2}+\delta f+f \delta$. Let $A$ be the corresponding non-commutative duplicate of $E$ obtained through the twisting tensor product, $A=k^{E} \otimes_{(f, \delta)}\left[k[X] /\left(X^{2}-X\right)\right]$.

Then $H^{*}(A)$ is finite dimensional if and only if the quiver of the set map $\varphi$ do not contains oriented cycles other than possible loops. In that case the Hochschild cohomology vanishes in degrees larger than 2.

Proof: The related quiver described in the previous section eliminates loops of $Q_{f}$ without creating oriented cycles, regardless of the coloration originated by the derivation.

Concerning cyclic homology we will use the results obtained in [7. Recall that the circuit associated to an oriented cycle $\gamma$ is the orbit of $\gamma$ through the natural action of the cyclic group of the same order than the length of $\gamma$. The set of circuits of length $j$ is denoted $\Theta_{j}$.

Note that in case of a proper cycle the action of the corresponding cyclic group is free on its orbit. We denote $\Omega_{a}$ the set of proper circuits of length $a$. The next result is proven in [7, p. 139]: 
Proposition 5.7. Let $Q$ be a quiver and $k$ be a field of characteristic zero.

For $n$ even

$$
\operatorname{dim} H C_{n}\left(\left(k Q_{2}\right)\right)=\left|\Theta_{n+1}\right|+\left|Q_{0}\right| .
$$

For $n$ odd

$$
\operatorname{dim} H C_{n}\left(\left(k Q_{2}\right)\right)=\sum_{2|a|(n+1)}\left|\Omega_{a}\right| .
$$

Theorem 5.8. Let $k$ be a field of characteristic zero, let $E$ be a finite set, let $A$ be a non-commutative duplicate algebra of $E$ and let $\varphi: E \rightarrow E$ be the set algebra map corresponding to the interlacing. The dimension of the even degree cyclic homology of $A$ is constant regardless of the derivation:

$$
\operatorname{dim} H C_{\text {even }}(A)=|E|+\mid \text { loops } \mid .
$$

Proof: We know that there are no odd length oriented cycles in the quiver of $f$, consequently the dimension is reduced to the number of vertices of the related quiver. Recall that each loop in the quiver of $f$ produces a new vertex in the related quiver.

In order to compute the odd cyclic homology, consider a 2-interlacing $(f, \delta)$ and the quiver $Q_{f}$ of $f$. Let $h(a)$ be the number of connected components of $Q_{f}$ containing a proper cycle of length $a$. We know that if $a$ is odd then $h(a)=0$.

Theorem 5.9. Let $k$ be a field of characteristic zero, let $E$ be a finite set, let $A$ be a non-commutative duplicate algebra of $E$ and let $f: E \rightarrow E$ be the set algebra map corresponding to the 2 -interlacing. Then for $n$ odd

$$
\operatorname{dim} H C_{n}(A)=\sum_{a \mid(n+1)} h(a) .
$$

Finally the Hochschild homology can also be obtained using the computation in [7] p. 140].

Theorem 5.10. Let $k$ be a field of characteristic different from 2, let $E$ be a finite set, let $A$ be a non-commutative duplicate algebra of $E$ and let $f: E \rightarrow E$ be the set algebra map corresponding to the 2-interlacing.

For $n$ odd

$$
\operatorname{dim} H H_{n}(A)=\sum_{a \mid(n+1)} h(a) .
$$

For $n$ even and $n \neq 0$

$$
\operatorname{dim} H H_{n}(A)=\sum_{a \mid n} h(a) .
$$

For $n=0$

$$
\operatorname{dim} H H_{0}(A)=|E|+\mid \text { loops } \mid .
$$




\section{REFERENCES}

[1] Baez, John C. Hochschild homology in a braided tensor category. Trans. Amer. Math. Soc. 344 (1994), no. 2, 885-906.

[2] Caenepeel, Stefaan; Militaru, Gigel; Zhu, Shenglin Frobenius and separable functors for generalized module categories and nonlinear equations. Lecture Notes in Mathematics, 1787, Springer-Verlag, Berlin, 2002.

[3] Brzeziński, Tomasz On modules associated to coalgebra Galois extensions. J. Algebra 215 (1999), no. 1, 290-317.

[4] Cap, Andreas; Schichl, Hermann; Vanžura, Jiří On twisted tensor products of algebras. Comm. Algebra 23 (1995), no. 12, 4701-4735.

[5] Cartier, Pierre Produits tensoriels tordus. Exposé au Séminaire des groupes quantiques de l'École Normale Supérieure, Paris, 1991-1992.

[6] Cibils, Claude Hochschild cohomology algebra of radical square zero algebras. Algebras and modules, II (Geiranger, 1996), 93-101, CMS Conf. Proc., 24, Amer. Math. Soc., Providence, RI, 1998.

[7] Cibils, Claude Cyclic and Hochschild homology of 2-nilpotent algebras. K-Theory 4 (1990), no. 2, 131-141.

[8] Majid, Shahn Physics for algebraists: noncommutative and noncocommutative Hopf algebras by a bicrossproduct construction. J. Algebra 130 (1990), no. 1, 17-64.

[9] Manin, Yu. I. Notes on quantum groups and quantum de Rham complexes. Teoret. Mat. Fiz. 92 (1992), no. 3, 425-450; translation in Theoret. and Math. Phys. 92 (1992), no. 3, 997-1023 (1993).

[10] Kassel, Christian Quantum groups. Graduate Texts in Mathematics, 155. Springer-Verlag, New York, 1995.

Université Montpellier 2, Institut de Mathématiques et de Modélisation de MontPellier, F-34095 Montpellier Cedex 5, France

E-mail address: Claude.Cibils@math.univ-montp2.fr 